\title{
A Case of Logopenic Variant of Primary Progressive Aphasia with Parkinsonism and Anosmia
}

\author{
Shoichi Sasaki ${ }^{\mathrm{a}, \mathrm{b}, *}$ \\ ${ }^{a}$ Department of Neurology, Agano City Hospital, Niigata, Japan \\ ${ }^{\mathrm{b}}$ Department of Neurology, Toyosaka Hospital, Niigata, Japan
}

Accepted 7 February 2020

\begin{abstract}
A 69-year-old right-handed woman developed difficulty naming objects and word-finding. The clinical features of language disorder and predominant atrophy on MRI and predominant hypoperfusion on ${ }^{123}$ I-IMP SPECT in the left temporoparietal junction area were consistent with the diagnostic criteria for the logopenic variant of primary progressive aphasia (lvPPA). Neurological examination showed slight right-side rigidity and resting tremor (UPDRS-III: 4). ${ }^{123}$ I-FP-CIT SPECT showed presynaptic dopamine transporter reduction in the posterior putamina with left-side predominance. The odor-stick identification test for Japanese exhibited complete loss of the sense of smell (anosmia). These findings suggest that lvPPA may be accompanied by parkinsonism and anosmia.
\end{abstract}

Keywords: Anosmia, ${ }^{123}$ I-FP-CIT SPECT, ${ }^{123}$ I-IMP SPECT, logopenic variant of primary progressive aphasia, magnetic resonance imaging, parkinsonism, primary progressive aphasia

\section{INTRODUCTION}

Primary progressive aphasia (PPA) consists of three main syndromes: progressive nonfluent aphasia, semantic dementia, and progressive logopenic aphasia. The logopenic variant of primary progressive aphasia (lvPPA), also known as logopenic progressive aphasia, is characterized by anomia, word-finding difficulties, impaired repetition of sentences and phrases, speech (phonologic) errors in spontaneous speech and naming, spared single-word comprehension and object knowledge, spared motor speech, and lack of frank agrammatism, and atrophy shown in the left posterior temporal cortex

\footnotetext{
${ }^{*}$ Correspondence to: Shoichi Sasaki, MD, PhD, Department of Neurology, Agano City Hospital, 13-23 Okayama-cho, Agano-shi, Niigata 959-2093, Japan. Tel.: +81 25062 2780; Fax: +81 25062 1598; E-mail: sasaki.shoichi@taupe.plala.or.jp.
}

and inferior parietal lobule [1, 2]. However, little is known about olfactory impairment and parkinsonism in patients with lvPPA. This report describes a case of lvPPA, accompanied by parkinsonism and the complete loss of the sense of smell (anosmia).

\section{CASE DESCRIPTION}

A 69-year-old right-handed woman with an educational attainment of 9 years developed a difficulty naming objects and word-finding approximately one and a half years before presenting at our hospital with a mild memory complaint such as forgetting an appointment and misplacing things. Her language comprehension was relatively well preserved. Clinical data of the patient are shown in Table 1. Mini-Mental State Examination (MMSE) indicated mild cognitive impairment (MMSE: 20/30) 
Table 1

Clinical data and results of linguistic function assessment

\begin{tabular}{lcc}
\hline Age at onset, y/gender & $69 / \mathrm{F}$ & \\
Education (y) & 9 & \\
Years of follow-up & 3 & \\
MMSE & 20 & \\
CDR & 0.5 & \\
UPDRS-III & 4 & \\
\hline Test of speech/language function & Raw score & Raw score \\
(Standard Language Test of & $\sim 18$ months & $\sim 30$ months \\
Aphasia) & after onset & after onset \\
\hline Confrontation naming & $7 / 20$ & $5 / 20$ \\
Word repetition & $10 / 10$ & $10 / 10$ \\
Sentence repetition & $3 / 5$ & $2 / 5$ \\
Single-word comprehension & $10 / 10$ & $9 / 10$ \\
Short sentence comprehension & $7 / 10$ & $6 / 10$ \\
Word reading (Kanji) & $3 / 5$ & $1 / 5$ \\
Word reading (Kana) & $5 / 5$ & $5 / 5$ \\
Short sentence reading & $5 / 5$ & $1 / 5$ \\
Word spelling (Kanji) & $0 / 5$ & $0 / 5$ \\
Word spelling (Kana) & $0 / 5$ & $0 / 5$ \\
Short sentence spelling & $0 / 5$ & $0 / 5$ \\
\hline
\end{tabular}

MMSE, Mini-Mental State Examination; CDR, Clinical Dementia Rating Scale; UPDRS, Unified Parkinson Disease Rating Scale.

and her Clinical Dementia Rating Scale (CDR) score was 0.5 . Language examination exhibited impaired single-word retrieval in spontaneous speech and naming, impaired repetition of sentences and phrases, spared single-word comprehension and object knowledge, spared motor speech and absence of frank agrammatism (Table 1). Forward digit span was 3 , whereas backward digit span was none. Neither apraxia of speech nor dysarthria was recognized. After one year, the results of linguistic assessment showed further deterioration of the language function (Table 1). Physical examination demonstrated no abnormalities, including of cardiac or pulmonary functions. On the neurological examination, the patient showed slight right-side rigidity and right-side predominant resting tremor (Unified Parkinson Disease Rating Scale (UPDRS)-III: 4) (Table 1). Since the parkinsonism was generally mild, medication was not required. The patient showed no prodromal symptoms, including REM sleep behavior disorder, constipation and orthostatic hypotension, nor atypical parkinsonian symptoms such as vertical supranuclear palsy, unsteadiness of gait, early falling, asymmetrical clumsiness of the limbs and marked rigidity-dystonia on the involved arm, which eliminates the possibility of progressive supranuclear palsy (PSP) or corticobasal syndrome (CBS). Laboratory examinations were in the normal range, including the results of complete blood count (CBC), comprehensive metabolic panel (CMP), and urinalysis. MRI revealed mild atrophy in the left posterior superior temporal and left inferior parietal junction regions (Fig. 1). The Voxel-based Specific Regional Analysis system for Alzheimer's Disease (VSRAD) Z-score ( $0 \sim 1$ : no atrophy, 1 2: mild atrophy, 2 3: moderate atrophy, 3 : severe atrophy) evaluating the degree of atrophy of medial temporal structures involving the entire region of the entorhinal cortex, hippocampus, and amygdala [3] was slightly elevated (VSRAD: 1.21), indicating mild medial temporal atrophy. After two years, medial temporal atrophy was deteriorated (VSRAD: 2.08). ${ }^{123}$ I-IMP SPECT (three-dimensional stereotactic surface projection: 3D-SSP) imaging showed marked hypoperfusion in the left temporo-parietal areas and the left frontal lobe, and to a lesser degree, in the posterior cingulate gyrus and precuneus predominantly on the left side (Supplementary Figure 1). ${ }^{123}$ I-FP-CIT dopamine transporter SPECT revealed a presynaptic dopamine transporter reduction in the posterior putamina with left-side predominance (an "egg shape" pattern) (Fig. 2). The odor-stick identification test for Japanese (OSIT-J) (Daiichi Yakuhin Sangyo, Tokyo) consisting of 12 daily odorants familiar to Japanese individuals (Japanese cypress, India ink, rose, perfume, cooking gas, menthol, sweaty socks, curry, Japanese orange, condensed milk, roasted garlic, and timber) showed a complete loss of the sense of smell (responses of odorless: anosmia) in both nostrils. Co-morbid medical conditions, such as chronic rhinitis, severe head injury, intracranial surgery, surgery in the nasal cavity, seasonal allergies, or other current respiratory infection, as well as heavy smoking were excluded.

\section{DISCUSSION}

In this case, linguistic impairment was insidious at the onset of the disease, gradually progressive, and initially characterized by a predominantly isolated language abnormality for more than 2 years, which is consistent with PPA [4]. Moreover, the clinical features of language disorder in the current case, such as impaired single-word retrieval in spontaneous speech and naming, impaired repetition of sentences and phrases, spared single-word comprehension and object knowledge, spared motor speech and absence of frank agrammatism, are consistent with the diagnostic criteria for lvPPA [2]. Additionally, both predominant atrophy on MRI and predominant hypoperfusion on SPECT in the left 

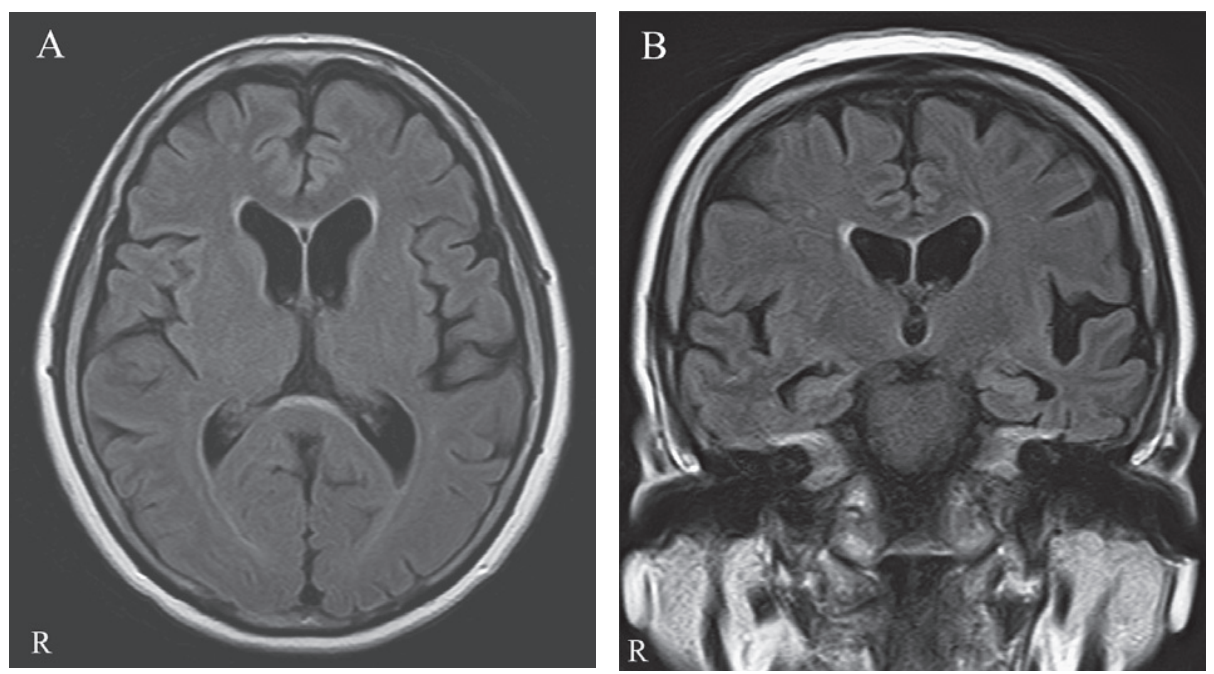

Fig. 1. The MRI shows mild focal atrophy in the left posterior superior temporal and left inferior parietal junction regions. A) Axial FLAIR image. B) Coronal FLAIR image.

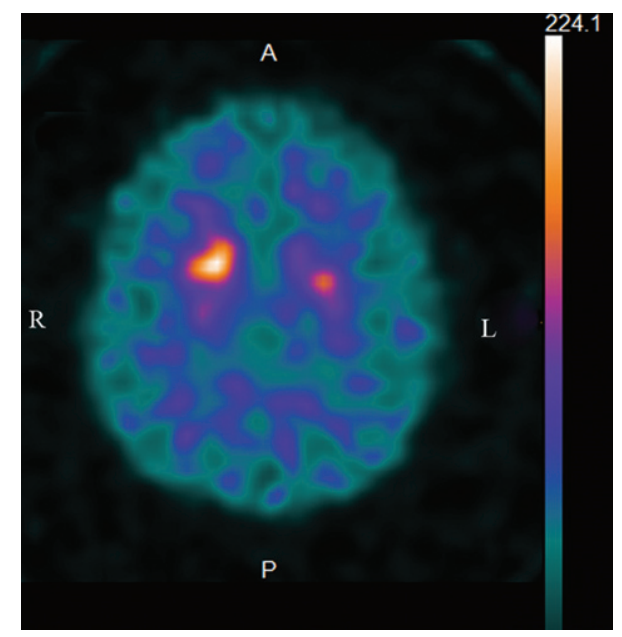

Fig. 2. ${ }^{123}$ I-FP-CIT dopamine transporter (DaTSCAN) SPECT reveals the presynaptic dopamine transporter reduction bilaterally in the posterior putamina with left-side predominance (the "egg shape" pattern).

temporo-parietal junction area are also compatible with the diagnostic criteria for lvPPA [2].

The present case initially presented with a language disorder, characterized by difficulty in finding words, sentence-repetition, and cognitive impairment including orientation, delayed recall and calculations. These findings are also consistent with Alzheimer's disease (AD). However, unlike typical AD, hippocampal regions in lvPPA are spared [5], which was also the case in our patient (VSRAD: 1.21). In typical AD patients, ${ }^{11} \mathrm{C}$-PIB and ${ }^{18}$ FFDG PET stud- ies show increased ${ }^{11} \mathrm{C}$-PIB binding in the frontal, temporal and parietal areas, and decreased cerebral glucose metabolism in the temporal and parietal regions, respectively [6], which resemble the findings in patients with lvPPA [7]. However, in contrast with typical $\mathrm{AD}$, which is characterized by bilateral, relatively symmetric temporoparietal hypometabolism, in lvPPA patients the degree of hypometabolism in the left parietal-temporal junction might be more severe and indeed, such severity can be an anatomical signature of damage due to lvPPA [7].

This case has two characteristic clinical manifestations, i.e., parkinsonism and anosmia. In regard with the first manifestation, parkinsonism is generally less frequent in IvPPA than in other variants of PPA. Rabinovici et al. [7] reported parkinsonism in 2 of 4 patients with lvPPA during a longitudinal followup, although the details of the parkinsonism were not described. Kremen et al. [8] reported that, in 28 patients with lvPPA, a pattern of specific extrapyramidal symptoms was seen, characterized only by an increased prevalence of gait disturbance (loss of balance or unsteadiness) (to approximately $20 \%$ of patients), a nonspecific neurologic sign that can be seen in conditions that do not involve extrapyramidal dysfunction, whereas extrapyramidal signs, which are more closely associated with parkinsonian syndromes, were seen less frequently (resting tremor: 0\%; bradykinesia: 0\%; rigidity: $10.7 \%$ ). Moreover, Graff-Radford et al. [9] reported that patients with lvPPA had fewer parkinsonian motor features than those with nonfluent/agrammatic aphasia, which was 
driven by bradykinesia, speech facial/expression, and rigidity. Gorno-Tempini et al. [2] suggested that a clear parkinsonian syndrome (rigidity, tremor) should not be present at time of diagnosis, although mild limb apraxia and difficulty with fine finger movement can be noted in PPA (especially in the nonfluent and semantic variants). Thus, it has been reported that parkinsonism symptoms such as resting tremor and rigidity are generally rare in lvPPA. These findings were supported by a study in which lvPPA patients had normal striatal ${ }^{123}$ I-FP-CIT uptake, in contrast to the reduced striatal tracer uptake observed in the patients with the non-fluent agrammatic variant of PPA prior to clinical parkinsonism [10]. In the present case study, parkinsonism with rigidity and resting tremor was observed (UPDRS-III: 4), and this observation was confirmed by the presynaptic dopamine transporter reduction in the bilateral posterior putamina (the "egg shape" pattern) on ${ }^{123}$ I-FP-CIT dopamine transporter SPECT, which is compatible with the initial stage of Parkinson's disease.

Regarding the second manifestation, i.e., the olfactory function in lvPPA, there has been a case report of a 58-year-old man with lvPPA, in whom the odor detection threshold was normal, although the cognitive threshold for scent recognition was significantly increased [11]. In terms of olfactory impairment in $\mathrm{AD}$ and parkinsonism, on the other hand, anosmia has been detected in approximately $40 \%$ of patients with Parkinson's disease (PD), $20 \%$ to $40 \%$ of those with $\mathrm{AD}$, and $65 \%$ of those with the Lewy body variant of $\mathrm{AD}$, and the incidence of anosmia in $\mathrm{PD}$ patients and AD patients increased with an increase in cognitive dysfunction [12, 13]. While the early changes associated with olfactory system processing have been reported to include decrements in the volume of the hippocampus, piriform cortex, anterior olfactory nucleus, and frontal poles of the brain $[14,15]$, in voxel-based morphometry in MRI, anosmia is associated with a significant decrease in gray matter volume in areas of the limbic system such as the hippocampus, parahippocampus, nucleus accumbens, and piriform cortex, which are correlated with olfaction-related structures [16]. Moreover, patients with mild cognitive impairment (MCI) or mild AD accompanied by parkinsonism showed more severe olfactory impairment compared to patients with MCI or mild AD without parkinsonism, or those with parkinsonism free of cognitive impairment [17]. In the present case with anosmia, the volume of medial temporal structures involving the entire region of the entorhinal cortex, hippocampus, and amygdala was slightly decreased (VSRAD: 1.21), followed by moderate atrophy (VSRAD: 2.08), as measured by MRI, which is in line with the previous reports $[16,18]$.

As for the pathomechanism of cognitive impairment and anosmia in lvPPA, there is a strong association between the clinical criteria of lvPPA and in vivo biomarkers such as PET-PIB positivity and decreased $A \beta_{42}$ and increased tau in the cerebrospinal fluid (CSF) [19-21], which is associated with the AD pathology, and in lvPPA, AD might be the most common underlying pathology $[7,20,21]$ in as many as $95 \%$ of cases [22]. Thus, lvPPA is defined as a variant of $\mathrm{AD}$. Olfactory dysfunction is one of the earliest clinical features or preclinical symptoms commonly observed and is associated with the pathogenesis of neurodegenerative diseases such as MCI [23] and AD $[24,25]$. In AD, olfactory identification impairment has been related to severity of illness; to both reduced hippocampal volume and decreased AD signature cortical thickness by MRI $[18,26]$; and to reduced blood oxygen level-dependent (BOLD) signals in the primary olfactory cortex, hippocampus, and insula regions by functional MRI [26]. Moreover, regarding the pathomechanism of parkinsonism and anosmia in lvPPA, olfactory dysfunction is also one of the earliest nonmotor features in $\mathrm{PD}$, and it precedes the onset of motor symptoms by years in that disorder [27, 28]. Olfactory impairment in PD seems to be primarily due to Lewy body pathology, including increased phosphorylated- $\alpha$-synuclein immunoreactivity in the olfactory bulb, particularly in the anterior olfactory nucleus $[29,30]$. Correlations are also observed between Brief Smell Identification Test scores and Lewy body pathology within the limbic and neocortical brain regions [31]. These findings of the reduced volume of the medial temporal structures in patients with $\mathrm{AD}$ and/or $\mathrm{PD}$ are compatible with those of the present lvPPA case with cognitive decline, parkinsonism and anosmia.

Thus, the coexistence of cognitive impairment (MCI or $\mathrm{AD}$ ) and parkinsonism may have been involved in the complete loss of the sense of smell (anosmia) in this lvPPA case. Moreover, bilateral anosmia, bilateral reduction of the presynaptic dopamine transporter with left-side predominance, and bilateral reduction of cerebral blood flow in the posterior cingulate gyrus with left predominance suggest that the lesion may have affected not only the left side, but also the limbic system and the nigrostriatal pathway on the right side in this case. 
In conclusion, to our knowledge this is a unique case in that it shows two characteristic clinical manifestations, i.e., parkinsonism and anosmia. Further studies are warranted to elucidate whether lvPPA is accompanied by parkinsonism and olfactory dysfunction in the clinical setting.

\section{ACKNOWLEDGMENTS}

The author would like to thank Ms. Fumiko Tsurumaki (a speech-language-hearing therapist) for her assistance with the Standard Language Test of Aphasia (SLTA).

\section{ETHICAL APPROVAL OF STUDIES AND INFORMED CONSENT}

The written informed consent was obtained from the patient and her son. The study was approved by the ethics committee of Agano City Hospital.

\section{CONFLICT OF INTEREST}

The author has no conflicts of interest.

\section{SUPPLEMENTARY MATERIAL}

The supplementary material is available in the electronic version of this article: http://dx.doi.org/ 10.3233/ADR-190158.

\section{REFERENCES}

[1] Gorno-Tempini ML, Dronkers NF, Rankin KP, Ogar JM, Phengrasamy L, Rosen HJ, Johnson JK, Weiner MW, Miller BL (2004) Cognition and anatomy in three variants of primary progressive aphasia. Ann Neurol 55, 335-346.

[2] Gorno-Tempini ML, Hillis AE, Weintraub S, Kertesz A, Mendez M, Cappa SF, Ogar JM, Rohrer JD, Black S, Boeve BF, Manes F, Dronkers NF, Vandenberghe R, Rascovsky K, Patterson K, Miller BL, Knopman DS, Hodges JR, Mesulam MM, Grossman M (2011) Classification of primary progressive aphasia and its variants. Neurology 76, 1006-1014.

[3] Matsuda H, Mizumura S, Nemoto K, Yamashita F, Imabayashi E, Sato N, Asada T (2012) Automatic voxelbased morphometry of structural MRI by SPM8 plus diffeomorphic anatomic registration through exponentiated lie algebra improves the diagnosis of probable Alzheimer disease. Am J Neuroradiol 33, 1109-1114.

[4] Mesulam MM (2001) Primary progressive aphasia. Ann Neurol 49, 425-432.

[5] Teichmann M, Kas A, Boutet C, Ferrieux S, Nogues M, Samri D, Rogan C, Dormont D, Dubois B, Migliaccio R (2013) Deciphering logopenic primary progressive aphasia: A clinical, imaging and biomarker investigation. Brain 136, 3474-3488.
[6] Edison P, Archer HA, Hinz R, Hammers A, Pavese N, Tai YF, Hotton G, Cutler D, Fox N, Kennedy A, Rossor M, Brooks DJ (2007) Amyloid, hypometabolism, and cognition in Alzheimer disease: An [11C]PIB and [18F]FDG PET study. Neurology 68, 501-508.

[7] Rabinovici GD, Jagust WJ, Furst AJ, Ogar JM, Racine CA, Mormino EC, O’Neil JP, Lal RA, Dronkers NF, Miller BL, Gorno-Tempini ML (2008) Abeta amyloid and glucose metabolism in three variants of primary progressive aphasia. Ann Neurol 64, 388-401.

[8] Kremen SA, Mendez MF, Tsai PH, Teng E (2011) Extrapyramidal signs in the primary progressive aphasias. Am J Alzheimers Dis Other Demen 26, 72-77.

[9] Graff-Radford J, Duffy JR, Strand EA, Josephs KA (2012) Parkinsonian motor features distinguish the agrammatic from logopenic variant of primary progressive aphasia. Parkinsonism Relat Disord 18, 890-892.

[10] Gil-Navarro S, Lomeña F, Cot A, Lladó A, Montagut N, Castellví M, Bosch B, Rami L, Antonell A, Balasa M, Pavia J, Iranzo A, Molinuevo JL, Sánchez-Valle R (2013) Decreased striatal dopamine transporter uptake in the nonfluent/agrammatic variant of primary progressive aphasia. Eur J Neurol 20, 1459-e126.

[11] Huihong Z, Pan W, Chunfeng Z, Yan W, Hui Z, Li C, Yuying $Z$ (2018) Olfactory and imaging features in atypical Alzheimer's disease. Transl Neurosci 9, 1-6.

[12] Tarakad A, Jankovic J (2017) Anosmia and ageusia in Parkinson's disease. Int Rev Neurobiol 133, 541-556.

[13] Olichney JM, Murphy C, Hofstetter CR, Foster K, Hansen LA, Thal LJ, Katzman R (2005) Anosmia is very common in the Lewy body variant of Alzheimer's disease. J Neurol Neurosurg Psychiatry 76, 1342-1347.

[14] Kemper T (1984) Neuroanatomical and neuropathological changes during age and dementia. In Clinical Neurology of Aging, Albert ML ed. Oxford University Press, New York, pp. 3-67.

[15] Wang J, You H, Liu JF, Ni DF, Zhang ZX, Guan J (2011) Association of olfactory bulb volume and olfactory sulcus depth with olfactory function in patients with Parkinson disease. AJNR Am J Neuroradiol 32, 677-681.

[16] Bitter T, Gudziol H, Burmeister HP, Mentzel HJ, GuntinasLichius O, Gaser C (2010) Anosmia leads to a loss of gray matter in cortical brain areas. Chem Senses 35, 407-415.

[17] Sasaki S (2019) Synergistic effects of Alzheimer's disease and parkinsonism on olfactory impairment. J Alzheimers Dis Parkinsonism 9, 2.

[18] Thomann PA, Dos Santos V, Seidl U, Toro P, Essig M, Schröder J (2009) MRI-derived atrophy of the olfactory bulb and tract in mild cognitive impairment and Alzheimer's disease. J Alzheimers Dis 17, 213-221.

[19] Gil-Navarro S, Lladó A, Rami L, Castellví M, Bosch B, Bargalló N, Lomeña F, Reñé R, Montagut N, Antonell A, Molinuevo JL, Sánchez-Valle R (2013) Neuroimaging and biochemical markers in the three variants of primary progressive aphasia. Dement Geriatr Cogn Disord 35, 106-117.

[20] Mesulam M, Wicklund A, Johnson N, Rogalski E, Léger GC, Rademaker A, Weintraub S, Bigio EH (2008) Alzheimer and frontotemporal pathology in subsets of primary progressive aphasia. Ann Neurol 63, 709-719.

[21] Bergeron D, Gorno-Tempini ML, Rabinovici GD, SantosSantos MA, Seeley W, Miller BL, Pijnenburg Y, Keulen MA, Groot C, van Berckel BNM, van der Flier WM, Scheltens P, Rohrer JD, Warren JD, Schott JM, Fox NC, SanchezValle R, Grau-Rivera O, Gelpi E, Seelaar H, Papma JM, van Swieten JC, Hodges JR, Leyton CE, Piguet O11, Rogalski 
EJ, Mesulam MM, Koric L, Nora K, Pariente J, Dickerson B, Mackenzie IR, Hsiung GR, Belliard S, Irwin DJ, Wolk DA, Grossman M, Jones M, Harris J, Mann D, Snowden JS, Chrem-Mendez P, Calandri IL, Amengual AA, Miguet-Alfonsi C, Magnin E, Magnani G, Santangelo R, Deramecourt V, Pasquier F, Mattsson N, Nilsson C, Hansson O, Keith J, Masellis M, Black SE, Matías-Guiu JA, Cabrera-Martin MN, Paquet C, Dumurgier J, Teichmann M, Sarazin M, Bottlaender M, Dubois B, Rowe CC, Villemagne VL, Vandenberghe R, Granadillo E, Teng E, Mendez M, Meyer PT, Frings L, Lleó A, Blesa R, Fortea J, Seo SW, Diehl-Schmid J, Grimmer T, Frederiksen KS, Sánchez-Juan P, Chételat G, Jansen W, Bouchard RW, Laforce RJ, Visser PJ, Ossenkoppele R (2018) Prevalence of amyloid- $\beta$ pathology in distinct variants of primary progressive aphasia. Ann Neurol 84, 729-740.

[22] Santos-Santos MA, Rabinovici GD, Iaccarino L, Ayakta N, Tammewar G, Lobach I, Henry ML, Hubbard I, Mandelli ML, Spinelli E, Miller ZA, Pressman PS, O’Neil JP, Ghosh P, Lazaris A, Meyer M, Watson C, Yoon SJ, Rosen HJ, Grinberg L, Seeley WW, Miller BL, Jagust WJ, GornoTempini ML (2018) Rates of amyloid imaging positivity in patients with primary progressive aphasia. JAMA Neurol 75, 342-352.

[23] Roberts RO, Christianson TJ, Kremers WK, Mielke MM, Machulda MM, Vassilaki M, Alhurani RE, Geda YE, Knopman DS, Petersen RC (2016) Association between olfactory dysfunction and amnestic mild cognitive impairment and Alzheimer disease dementia. JAMA Neurol 73, 93-101.
[24] Doty RL, Reyes PF, Gregor T (1987) Presence of both odor identification and detection deficits in Alzheimer's disease. Brain Res Bull 18, 597-600.

[25] Tabert MH, Liu X, Doty RL, Serby M, Zamora D, Pelton GH, Marder K, Albers MW, Stem Y, Devanand DP (2005) A 10-item smell identification scale related to risk for Alzheimer's disease. Ann Neurol 58, 155-160.

[26] Velayudhan L (2015) Smell identification function and Alzheimer's disease: A selective review. Curr Opin Psychiatry 28, 173-179.

[27] Doty RL (2012) Olfactory dysfunction in Parkinson disease. Nat Rev Neurol 8, 329-339.

[28] Ross GW, Petrovitch H, Abbott RD, Tanner CM, Popper J, Masaki K, Launer L, White LR (2008) Association of olfactory dysfunction with risk for future Parkinson's disease. Ann Neurol 63, 167-173.

[29] Braak H, Rub U, Gai WP, Del Tredici K (2003) Idiopathic Parkinson's disease: possible route by which vulnerable neuronal types may be subject to neuroinvasion by an unknown pathogen. J Neural Transm 119, 517-536.

[30] Zapiec B, Dieriks BV, Tan S, Faull RLM, Mombaerts P, Curtis MA (2017) A ventral glomerular deficit in Parkinson's disease revealed by whole olfactory bulb reconstruction. Brain 140, 2722-2736.

[31] Wilson RS, Yu L, Schneider JA, Arnold SE, Buchman AS, Bennett DA (2011) Lewy bodies and olfactory dysfunction in old age. Chem Senses 36, 367-373. 